\title{
Adult small bowel Dieulafoy lesion
}

\author{
A Fox, K Ravi, P C Leeder, B J Britton, B F Warren
}

\begin{abstract}
A 47 year old woman presented with melaena and haemodynamic instability. Preliminary investigations failed to locate the source of bleeding. At laparotomy an arteriovenous malformation was identified in the distal ileum. Histology revealed this to be of the Dieulafoy type. This is the first published case of a histologically proved ileal Dieulafoy lesion in an adult. (Postgrad Med f 2001;77:783-784)
\end{abstract}

Keywords: Dieulafoy lesion; arteriovenous malformation; small bowel

The Dieulafoy lesion as a rare cause of gastrointestinal haemorrhage was first identified by Gallard in 1884 and formally described by Dieulafoy in $1897 .^{12}$ Macroscopically this arteriovenous malformation (AVM) comprises a small pea sized lesion appearing as a mucosal defect with an artery protruding from its base. ${ }^{3}$ Histologically, a thick walled arterial vessel is seen. This is larger than surrounding submucosal vessels, and runs below the muscularis mucosae. Similar appearances have been reported throughout the gastrointestinal tract. ${ }^{3}$ Although the pathogenesis is unclear, the lesion is believed to be congenital in origin. ${ }^{4}$

Dieulafoy lesions give rise to $0.3 \%-1.5 \%$ of major gastrointestinal bleeds. Most commonly, this would present as haematemesis $(28 \%)$ or melaena $(18 \%) .^{5}$ Dieulafoy lesions are typically found in the stomach (within $6 \mathrm{~cm}$ of the gastro-oesophageal junction) on the lesser curve. ${ }^{6}$ Small bowel Dieulafoy lesions are very

Department of Upper Gastrointestinal and Hepatobiliary Surgery, John Radcliffe Hospital Headington, Oxford, UK

A Fox

K Ravi

P C Leeder

B J Britton

B F Warren

Correspondence: Dr Andreas Fox, Department of Surgery, Leicester General Hospital, Gwendolen Road, Leicester LE5 4PW, UK

andreasfox@hotmail.com

Submitted 18 May 2001 Accepted 27 July 2001

Figure 1 Dieulafoy vascular malformation of the ileum. The lesion is shown in its entirety with thrombosis within, and evidence of bleeding on the luminal surface. It is surrounded by normal small bowel mucosa. rare $^{7}$ and are most commonly seen in the jejunum. ${ }^{7}$ In around $5 \%$ of gastrointestinal bleeds, preliminary investigations (endoscopy, barium studies, colonoscopy) fail to reveal the source of bleeding. ${ }^{8}$

\section{Case report}

A 47 year old woman presented with melaena, requiring multiple blood transfusions. She had no past history or symptoms of gastrointestinal dysfunction and her bowel habit was previously normal. She had not experienced any recent loss of weight or appetite. She was a known hypertensive well controlled on medication (felodipine $5 \mathrm{mg}$ daily).

On admission, she was tachycardic (heart rate 112 beats/min regular) and had significant hypotension (blood pressure 98/65 $\mathrm{mm} \mathrm{Hg}$ ). Her haemoglobin concentration was $86 \mathrm{~g} / \mathrm{l}$, with a normal mean corpuscular volume and no clotting derangements. An upper gastrointestinal endoscopy was performed which did not show any abnormality. Flexible sigmoidoscopy was unsuccessful due to a large amount of blood in the rectum. She continued to bleed and had a further episode of hypotension. It was decided that angiography would delay much needed surgery and so exploratory laparotomy was performed to locate the source of bleeding.

Access was through a lower midline incision. The entire colon was full of blood as were the distal $90 \mathrm{~cm}$ of the ileum, with a sharp proximal cut off point. Approximately $7.5 \mathrm{~cm}$ distal to this, a polypoid lesion was felt on external palpation of the ileum. Careful palpation of the rest of the small bowel failed to reveal any other lesions or diverticulae. Enterotomy was performed and the lesion was confirmed to be actively bleeding after isolating the small bowel between non-crushing clamps. A limited small bowel resection and primary anastomosis was performed. No colonic lesion was identified after extensive lavage. The patient made a rapid and uncomplicated recovery with no further bleeds.

Macroscopic examination revealed a vascular malformation $7 \times 5 \times 5 \mathrm{~mm}$ in diameter with a visible blood clot within. Microscopy revealed a lesion comprising of thick walled arteries and veins representing an AVM. There was a degree of thrombosis and recanalisation. The appearances were those of an AVM of the Dieulafoy type (fig 1).

\section{Discussion}

Between $75 \%$ and $95 \%$ of cases of Dieulafoy lesions have been reported to occur in the stomach. ${ }^{6}$ Reports of extragastric Dieulafoy 
lesions have been divided in the literature between those found in the oesophagus, duodenum, ${ }^{3}$ jejunum, ${ }^{3}$ ileum, ${ }^{910}$ and the large bowel and rectum. ${ }^{3}$

The true incidence is unknown. There is a male to female ratio of $2: 1$ and the median age at presentation is 54 years (range $50-60$ years). The extragastric lesions are seen more commonly in the large than the small bowel. Patients present with haemodynamic instability, as chronic or recurrent gastrointestinal bleeding. Such patients pose a diagnostic dilemma with preliminary investigations often being normal or inconclusive. The diagnosis is made at surgery or after more specialised investigations have been performed.

Rarely do features of the history point to the obscure cause. Review of all relevant past investigations is important, and aggressive resuscitation should parallel any further investigations. In the case of a massive bleed and a compromised patient, laparotomy becomes mandatory. Although it is not always possible to identify AVMs at laparotomy, intraoperative localisation methods include careful palpation, transillumination, and enteroscopy. ${ }^{7}{ }^{11}$

In the case of a stable or recurrent gastrointestinal bleed, a variety of investigations have been suggested. The small bowel still remains difficult to visualise directly on routine endoscopy. The advent of push enteroscopy is providing a solution for the jejunum, with encouraging results. ${ }^{8}$ Selective angiography yielded the best results in a patient who was actively bleeding. ${ }^{11}$ Small vascular abnormalities may still pass unrecognised and it is difficult to exclude any other causes of bleeding. Isotope scanning techniques have also been described but tend to be less in favour, giving high false positive and negative results with poor specificity. ${ }^{11}$ Dye staining techniques for easier identification of the involved segment of bowel at laparotomy have also been used. ${ }^{7}$

The principles of management of Dieulafoy lesions depend on their location. With gastric, duodenal and proximal jejunal lesions, endoscopic therapy achieves permanent haemostasis in $85 \%$ of cases with only $5 \%$ of cases proceeding to surgery. ${ }^{9}$ Both thermal and injection modalities have been used successfully; however, the optimal endoscopic treatment has not yet been determined. ${ }^{5}$ In proximal small bowel, surgical exploration with intraoperative endoscopy have yielded excellent results and avoids resection. ${ }^{2}$

We have identified two reports of ileal Dieulafoy lesions. Raijman and Haber describe a 72

\section{Learning points}

- The Dieulafoy lesion is an arteriovenous malformation typically found in the stomach.

- Extragastric lesions are rare, and an uncommon cause of gastrointestinal bleeds.

- The investigation and management of lower gastrointestinal bleeding poses an important diagnostic problem, following the elimination of common causes. The lesion should be suspected in such cases.

- There is lack of consensus as to the optimum type and order of investigations in obscure causes of gastrointestinal bleeds.

year old man who had experienced chronic lower gastrointestinal bleeding and had had multiple previous investigations. ${ }^{9}$ They identified an apparent Dieulafoy lesion on a lower gastrointestinal endoscopy with a coincidental surgical clip protruding from its base. There is no histological evidence in their report. Chen details a case report of a 15 year old boy who presented with melaena, clinical features of anaemia, and haemoglobin drop. ${ }^{10}$ The lesion was identified on mesenteric arteriography and confirmed on histology. We describe here a case of an adult ileal Dieulafoy lesion with histological evidence.

1 Dieulafoy P. Lecons clinique de l'Hotel de Paris. Paris: Mason, 1897;2:1.

2 Al-Mishlab T, Amin AM, Ellul JPM. Dieulafoy's lesion: an obscure cause of GI bleeding. F R Coll Surg Edinb 1999;44: $222-5$.

3 Gadenstätter M, Wetscher G, Crookes PF, et al. Dieulafoy's disease of the large and small bowel. $f$ Clin Gastroenterol 1998;27:169-172.

4 Vetto JT, Richmond PS, Karriger K, et al. Cirsoid aneurysm of the jejunum: an unrecognised cause of massive gastroof the jejunum: an unrecognised cause of massire
intestinal bleeding. Arch Surg 1989;124:1460-2.

5 Reilly HF III, Al-Kawas FH. Dieulafoy's lesion: diagnosis and management. Dig Dis Sci 1991;36:1702-7.

6 Veldhuyzen van Zanten ST, Bartelsman JF, Schipper ME, et al. Recurrent massive hematemesis from Dieulafoy vascular malformation: review of 101 cases. Gut 1986;27:213-22.

7 Goins WA, Chatman DM, Kaviani MJ. Massive lower gastrointestinal bleeding due to "Dieulafoy's vascular malformation" of the jejunum: case report. F Natl Med Assoc 1995;87:766-70.

8 Descamps C, Schmit A, Van Gossum A. "Missed" upper gastrointestinal tract lesions may explain "occult" bleeding. Endoscopy 1999;31:452-5.

9 Raijman I, Haber GB. Small intestine Dieulafoy lesion: or "Dieulaclip"? Endoscopy 1995;27:215.

10 Chen KT. Intestinal bleeding from a caliber persistent submucosal artery in the ileum. F Clin Gastroenterol 1985;7: mucosal

11 Spiller RC, Parkins RA. Recurrent gastrointestinal bleeding Spiller RC, Parkins RA. Recurrent gastrointestinal bleeding
of obscure origin: report of 17 cases and a guide to logical management. Br f Surg 1983;70:489-93. 